\title{
INVERSE LIMIT SEQUENCES WITH COVERING MAPS
}

\author{
BY \\ M. C. McCORD $\left({ }^{1}\right)$
}

1. Introduction. The purpose of this paper is two-fold. We define a class of spaces called solenoidal spaces, which generalize the solenoids of van Dantzig [13], and study their structure $(\$ \$ 4,5)$. Then we use part of the structure developed to prove a theorem on the homogeneity of certain solenoidal spaces (\$6). A solenoidal space is the limit of an inverse limit sequence of "nice" spaces (the precise definition is below) where the bonding maps are regular covering maps $\left({ }^{2}\right)$. We will see that these spaces still have many of the properties of the classical solenoids.

A space $X$ is called homogeneous if for each pair $x, y$ of points of $X$ there is a homeomorphism of $(X, x)$ onto $(X, y)$. M. K. Fort, Jr. [8] asked the general question, "When is an inverse limit space homogeneous?" The pseudo-arc, which is known to be homogeneous (see Bing [1]), can be described roughly as an inverse limit of arcs where the bonding maps become sufficiently "crooked." Perhaps one can obtain some result on homogeneity in which "crookedness" of the bonding maps is one of the assumptions. (See Brown [4] for a precise definition of $\epsilon$-crooked map.) Probably one should restrict himself to the case where the factor spaces are 1-dimensional, for the following reason. Brown [4] has shown that an inverse limit of locally connected continua with sufficiently crooked bonding maps is hereditarily indecomposable; and Bing [2] has shown that if $X$ is an $n$-dimensional, hereditarily indecomposable continuum and $n>1$, then $X$ is not homogeneous. The word "probably" was used two sentences ago because of the fact that dimension may be lowered by taking an inverse limit of continua with "onto" bonding maps (although it can never be raised).

The theorem in $\$ 6$ goes in the opposite direction from the preceding suggestion, by assuming that the bonding maps are "smooth." The assumption of local smoothness in the sense of differentiability will of course do no good. A kind of global smoothness is needed; this is why covering maps are appropriate. Case [5] has taken an inverse limit of universal curves where the bonding maps are regular covering maps to get a new example of a 1-dimensional homogeneous continuum containing arcs. Two of our theorems almost generalize two of his, but, as they stand, do not imply his.

For notation and terminology on inverse limit sequences refer to [7]. We

Received by the editors July $12,1963$.

(1) This research was partially supported by the Air Force under SAR G AF AFOSR 62-20.

${ }^{2}$ ) In [14] van Heemert dealt with inverse limits of manifolds where the bonding maps are covering maps. 
consider only the case where the directed set of indices is the positive integers. If $(X, f)$ is an inverse limit sequence, the maps $f_{m}^{n}: X_{n} \rightarrow X_{m}(m \leqq n)$ are called bonding maps. If $x \in X_{\infty}$, then $x_{n}$ will often be used to denote the $n$th coordinate $f_{n}(x)$ of $x$. The symbol | will be used to indicate the ends of proofs.

2. Motivation: The $P$-adic solenoid. If $P=\left(p_{1}, p_{2}, \ldots\right)$ is a sequence of prime numbers ( 1 not being included as a prime), the $P$-adic solenoid $\Sigma_{P}$ is defined as the limit of the inverse limit sequence $(X, f)$, where for each $n, X_{n}=\{z:|z|=1\}$ (unit circle in the complex plane), and where each bonding map $f_{n}^{n+1}: X_{n+1} \rightarrow X_{n}$ is given by $f_{n}^{n+1}(z)=z^{p^{n}}$. Call prime sequences $P$ and $Q$ equivalent (written $P \sim Q$ ) if a finite number of terms can be deleted from each sequence so that every prime number occurs the same number of times in the deleted sequences. Bing [3] remarked that if $P \sim Q$ then $\Sigma_{P}$ is homeomorphic to $\Sigma_{Q}$ and suggested, "Perhaps the converse of this is true." One can see that $P \sim Q$ if and only if $\Sigma_{P}$ is homeomorphic to $\Sigma_{Q}$ (written $\Sigma_{P} \equiv \Sigma_{Q}$ ) as follows $\left({ }^{3}\right)$ : From the continuity theorem for Cech cohomology $\left[7\right.$, p. 261] one sees that $H^{1}\left(\Sigma_{P}\right)$ is isomorphic to the group $F_{P}$ of $P$-adic raionals (all rationals of the form $k /\left(p_{1} p_{2} \cdots p_{n}\right)$ where $k$ is an integer and $n$ is a positive integer). Also it can be seen that $\Sigma_{P}$, as a topological group, is topologically isomorphic to the character group of $F_{P}$ (written $\Sigma_{P} \approx_{\text {top }} \hat{F}_{P}$ ). By number-theoretic considerations one can see that $F_{P}$ is isomorphic to $F_{Q}$ (written $F_{P} \approx F_{Q}$ ) if and only if $P \sim Q$. Thus

$$
\Sigma_{P} \equiv \Sigma_{Q} \Longrightarrow H^{1}\left(\Sigma_{P}\right) \approx H^{1}\left(\Sigma_{Q}\right) \Longrightarrow F_{P} \approx F_{Q} \Longrightarrow P \sim Q .
$$

Conversely,

$$
P \sim Q \Longrightarrow F_{P} \approx F_{Q} \Longrightarrow \hat{F}_{P} \approx_{\text {top }} \hat{F}_{Q} \Longrightarrow \Sigma_{P} \approx \text { top } \Sigma_{Q}
$$

3. Solenoidal spaces. For basic notions of covering space theory refer to [9] or [11].

DEFinition 3.1. A solenoidal sequence is an inverse limit sequence $(X, f)$ such that (1) each space $X_{n}$ is nice in the sense that it is connected, locally pathwise connected, and semi-locally simply connected, and (2) each bonding map $f_{1}^{n}: X_{n} \rightarrow X_{1}$ is a regular covering map. The limit $X_{\infty}$ will be called a solenoidal space.

REMARK 3.2. The spaces $X_{n}$ are assumed to be nice in order to guarantee the constructions of covering space theory. In particular, they could be polyhedra.

REMARK 3.3. Condition (2) implies that each bonding map $f_{m}^{n}: X_{n} \rightarrow X_{m}$ $(m \leqq n)$ is a regular covering map.

REMARK 3.4. If each $X_{n}$ is a continuum then $X_{\infty}$ is a continuum.

( ${ }^{3}$ Essentially the same result was stated by van Dantzig. 
EXAmple 1. For each $n$, let $X_{n}$ be the $r$-dimensional torus $T^{r}=S^{1} \times \cdots$ $\times S^{1}$ ( $r$ times). Take each $f_{n}^{n+1}: X_{n+1} \rightarrow X_{n}$ to be of the form $f_{n}^{n+1}\left(z_{1}, \cdots, z_{r}\right)=$ $\left(z_{1}^{p_{1}}, \cdots, z_{r}^{p_{r}}\right)$, where the $p_{i}$ 's are positive integers.

ExAMPLE 2. For each $n, X_{n}$ is obtained as follows. Take two disjoint copies of $S^{1}$ and identify them at the $2^{n-1}$ points $\exp \left(2 \pi i k / 2^{n-1}\right), k=0, \cdots, 2^{n-1}-1$. ( $X_{1}$ is a figure eight.) Then the map $s: S^{1} \rightarrow S^{1}$ given by $s(z)=z^{2}$ induces a covering map $f_{n}^{n+1}: X_{n+1} \rightarrow X_{n}$. The regularity of $f_{1}^{n}$ follows from the fact that its covering transformations act transitively on the fibers.

EXAMPLE 3. If $Y_{1}$ is any nice space, $F_{1}$ is the fundamental group $\pi\left(Y_{1}, b_{1}\right)$, and $\left(F_{2}, F_{3}, \ldots\right)$ is a decreasing sequence of normal subgroups of $F_{1}$, then we can construct a solenoidal sequence $(Y, g)$ with base points $b_{n}$ in $Y_{n}$ such that

$$
\left(g_{1}^{n}\right)_{*}\left(\pi\left(Y_{n}, b_{n}\right)\right)=F_{n} .
$$

Example 4. As a special case of the method of Example 3, we obtain a solenoidal sequence of closed 3-manifolds as follows. Let $(X, f)$ be as in Example 2. Let $Y_{1}$ be the connected sum of the 3-manifold $S^{1} \times S^{2}$ with itself. Then $F_{1}=\pi\left(Y_{1}, b_{1}\right)$ is a free group on two generators, hence isomorphic to $\pi\left(X_{1}, 1\right)$. The decreasing sequence $\left(\left(f_{1}^{n}\right)_{\star} \pi\left(X_{n}, 1\right)\right), n=2, \cdots$, of normal subgroups of $\pi\left(X_{1}, 1\right)$ then defines a decreasing sequence $\left(F_{2}, F_{3}, \ldots\right)$ of normal subgroups of $F_{1}$. Hence we may obtain $(Y, g)$. One might say that the solenoidal sequence $(X, f)$ of 1-polyhedra serves as a model for constructing the solenoidal sequence $(Y, g)$ of 3 -manifolds.

4. A lemma on covering transformations. The result of this section will be used twice in $\S 5$. Suppose we are given a commutative diagram

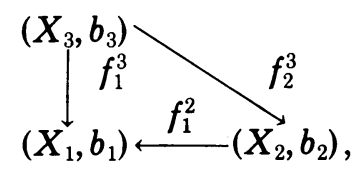

where the three maps are regular covering maps and the base points $b_{k}$ are fixed throughout the discussion. Let $F_{1}$ be the fundamental group $\pi\left(X_{1}, b_{1}\right)$ and for $k=2,3$ let $F_{k}=\left(f_{1}^{k}\right)_{*}\left(\pi\left(X_{k}, b_{k}\right)\right)$. Thus $F_{1} \supset F_{2} \supset F_{3}$ and $F_{2}, F_{3}$ are normal in $F_{1}$. Let $G_{k}$ be the covering transformation group of $f_{1}^{k}$. There is a canonical isomorphism $\phi_{k}$ of $Q_{k}=F_{1} / F_{k}$ onto $G_{k}$. Since $F_{3} \subset F_{2}$ there is a natural homomorphism $\nu: Q_{3} \rightarrow Q_{2}$ (given by $\nu\left(a F_{3}\right)=a F_{2}$ ). Now define $\mu: G_{3} \rightarrow G_{2}$ by commutativity in

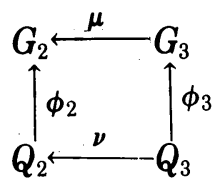

Lemma 4.1. (a) If $g_{3} \in G_{3}$ and $g_{2}=\mu\left(g_{3}\right)$ then the diagram 


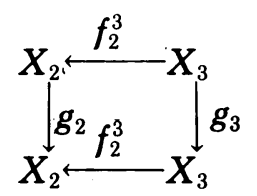

is commutative. (b) If $g_{2} \in G_{2}, g_{3} \in G_{3}$, and the diagram (4.3) is commutative at some point $x_{3} \in X_{3}$, then $g_{2}=\mu\left(g_{3}\right)$.

Proof. (a) Let $h_{3}=\left[\alpha_{1}\right] F_{3}$ be the element of $Q_{3}$ such that $\phi_{3}\left(h_{3}\right)=g_{3}$ (where $\alpha_{1}$ is a loop on $\left.b_{1}\right)$, and let $h_{2}=\nu\left(h_{3}\right)=\left[\alpha_{1}\right] F_{2}$. By commutativity of (4.2) we have $\phi_{2}\left(h_{2}\right)=g_{2}$. Now lift $\alpha_{1}$ by $f_{1}^{2}$ to the path $\alpha_{2}$ starting at $b_{2}$. Then from the definition of $\phi_{2}, g_{2}$ is the unique element of $G_{2}$ such that $g_{2}\left(b_{2}\right)=\alpha_{2}(1)$. Lift $\alpha_{2}$ by $f_{2}^{3}$ to the path $\alpha_{3}$ starting at $b_{3}$. But $f_{1}^{3} \alpha_{3}=f_{1}^{2} f_{2}^{3} \alpha_{3}=f_{1}^{2} \alpha_{2}=\alpha_{1}$, so that $g_{3}=\phi_{3}\left(h_{3}\right)$ satisfies $g_{3}\left(b_{3}\right)=\alpha_{3}(1)$. We conclude that $f_{2}^{3} g_{3}\left(b_{3}\right)=f_{2}^{3} \alpha_{3}(1)$ $=\alpha_{2}(1)=g_{2}\left(b_{2}\right)$.

Now take an arbitrary point $x_{3}$ in $X_{3}$ and let $x_{2}=f_{2}^{3}\left(x_{3}\right)$. We can determine $g_{2}\left(x_{2}\right)$ and $g_{3}\left(x_{3}\right)$ as follows. Take a path $\beta_{3}$ from $b_{3}$ to $x_{3}$, and let $\beta_{2}=f_{2}^{3} \beta_{3}$ and $\beta_{1}=f_{1}^{2} \beta_{2}=f_{1}^{3} \beta_{3}$. Since $\beta_{2}$ is a path from $b_{2}$ to $x_{2}$ we see (e.g. from [11, p. 196]) that if we lift $\beta_{1}$ by $f_{1}^{2}$ to the path $\beta_{2}^{\prime}$ starting at $g_{2}\left(b_{2}\right)$, then $g_{2}\left(x_{2}\right)$ $=\beta_{2}^{\prime}(1)$. Since $f_{2}^{3} g_{3}\left(b_{3}\right)=g_{2}\left(b_{2}\right)$ (by the preceding paragraph) we may lift $\beta_{2}^{\prime}$ by $f_{2}^{3}$ to the path $\beta_{3}^{\prime}$ starting at $g_{3}\left(b_{3}\right)$. Then, since $f_{1}^{3} \beta_{3}^{\prime}=\beta_{1}=f_{1}^{3} \beta_{3}$, we have $g_{3}\left(x_{3}\right)=\beta_{3}^{\prime}(1)$. Thus $f_{2}^{3} g_{3}\left(x_{3}\right)=f_{2}^{3} \beta_{3}^{\prime}(1)=\beta_{2}^{\prime}(1)=g_{2}\left(x_{2}\right)$, which shows the commutativity of (4.3).

(b) We are supposing that at some point $x_{3}, g_{2} f_{2}^{3}\left(x_{3}\right)=f_{2}^{3} g_{3}\left(x_{3}\right)$. By part (a), then, $g_{2} f_{2}^{3}\left(x_{3}\right)=\mu\left(g_{3}\right) f_{2}^{3}\left(x_{3}\right)$. But an element in $G_{2}$ is determined by its value on a single point, so that $g_{2}=\mu\left(g_{3}\right)$.

REMARK 4.2. This lemma shows that the definition of $\mu$ is independent of the choice of the base points $b_{k}$.

5. The structure of solenoidal spaces. We assume in this section that we are given an arbitrary solenoidal sequence $(X, f)$. To avoid triviality we assume that for each $n$ the covering $f_{n}^{n+1}: X_{n+1} \rightarrow X_{n}$ is $k_{n}$-to-1 where $k_{n}$ is a cardinal greater than 1.

Let us choose once and for all a base point $b=\left(b_{1}, b_{2}, \ldots\right)$ in $X_{\infty}$. For each $n$, let $F_{n}=\left(f_{1}^{n}\right)_{*}\left(\pi\left(X_{n}, b_{n}\right)\right)$ so that we have a descending sequence of groups $F_{1} \supset F_{2} \supset \cdots$, each $F_{n}$ being normal in $F_{1}=\pi\left(X_{1}, b_{1}\right)$. Let $Q_{n}=$ $F_{1} / F_{n}$ and let $\phi_{n}$ be the canonical isomorphism of $Q_{n}$ onto the covering transformation group $G_{n}$ of $f_{1}^{n}: X_{n} \rightarrow X_{1}$. Defining homomorphisms $\nu_{n}^{n+1}: Q_{n+1} \rightarrow Q_{n}$ and $\mu_{n}^{n+1}: G_{n+1} \rightarrow G_{n}$ according to the prescription of the preceding section, we get inverse limit sequences of groups $(Q, \nu)$ and $(G, \mu)$ with limit groups $Q_{\infty}$ and $G_{\infty}$. Since from the definition of $\mu_{n}^{n+1}, \phi_{n} \nu_{n}^{n+1}=\mu_{n}^{n+1} \phi_{n+1}$, the sequence $\left(\phi_{n}\right)$ induces an isomorphism $\phi_{\infty}: Q_{\infty} \rightarrow G_{\infty}$. Consider each $G_{n}$ as a discrete topological group, and give $G_{\infty}$ the inverse limit topology.

Lemma 5.1. $G_{\infty}$ is totally disconnected and perfect. If the coverings $f_{n}^{n+1}$ are 
finite-to-one then $G_{\infty}$ is homeomorphic to the Cantor set.

Proof. It is easy to see that an inverse limit of totally disconnected spaces is totally disconnected. From the fact that for each $g_{n}$ in $G_{n},\left(\mu_{n}^{n+1}\right)^{-1}\left(g_{n}\right)$ contains $k_{n}>1$ elements, one can see that $G_{\infty}$ is perfect. If each $k_{n}$ is finite, then each $G_{n}$ is finite, so that $G_{\infty}$ is compact metric and is therefore homeomorphic to the Cantor set. I

Lemma 5.2. $G_{\infty}$ acts on $X_{\infty}$ as an effective topological transformation group.

Proof. Suppose $g=\left(g_{1}, g_{2}, \cdots\right) \in G_{\infty}$. By Lemma 4.1 we have for each $n$ the commutativity relation $g_{n} f_{n}^{n+1}=f_{n}^{n+1} g_{n+1}$, so that $g$ induces a homeomorphism of $X_{\infty}$ onto itself, which we still denote by $g$, i.e., for

$$
x=\left(x_{1}, x_{2}, \cdots\right) \in X_{\infty},
$$

$g(x)=\left(g_{1}\left(x_{1}\right), g_{2}\left(x_{2}\right), \ldots\right)$. Obviously we have (1) $\left(g \cdot g^{\prime}\right)(x)=g\left(g^{\prime}(x)\right)$ and (2) the identity element of $G_{\infty}$ is the unique element of $G_{\infty}$ which acts as the identity transformation on $X_{\infty}$. Now we want to show that the map $G_{\infty} \times X_{\infty} \rightarrow X_{\infty}$ given by $(g, x) \rightarrow g(x)$ is continuous. Suppose $\left(g^{0}, x^{0}\right)$ is given and $U$ is a neighborhood of $g^{0}\left(x^{0}\right)$. By $[7$, p.218] we may assume that $U=f_{n}^{-1}\left(U_{n}\right)$ where $U_{n}$ is a neighborhood of $g_{n}^{0}\left(x_{n}^{0}\right)$. Since $G_{n}$ is discrete, $V=\mu_{n}^{-1}\left(g_{n}^{0}\right)$ is a neighborhood of $g^{0}$. Since $g_{n}^{0} f_{n}$ is continuous, there is a neighborhood $W$ of $x^{0}$ such that $g_{n}^{0} f_{n}(W) \subset U_{n}$. Then if $(g, x) \in V \times W$, $f_{n} g(x)=g_{n} f_{n}(x)=g_{n}^{0} f_{n}(x) \in U_{n}$, so that $g(x) \in U$. I

Lemma 5.3. If $x$ and $x^{\prime}$ are in $X_{\infty}$ and $f_{1}(x)=f_{1}\left(x^{\prime}\right)$, then there is one and only one $g$ in $G_{\infty}$ such that $g(x)=x^{\prime}$.

Proof. Let $x=\left(x_{1}, x_{2}, \ldots\right)$ and $x^{\prime}=\left(x_{1}^{\prime}, x_{2}^{\prime}, \ldots\right)$ where $x_{1}=x_{1}^{\prime}$. Since $f_{1}^{n}\left(x_{n}\right)=f_{1}^{n}\left(x_{n}^{\prime}\right)$ and $f_{1}^{n}$ is regular, there is a unique $g_{n} \in G_{n}$ such that $g_{n}\left(x_{n}\right)$ $=x_{n}^{\prime}$. Since

$$
g_{n} f_{n}^{n+1}\left(x_{n+1}\right)=g_{n}\left(x_{n}\right)=x_{n}^{\prime}=f_{n}^{n+1}\left(x_{n+1}^{\prime}\right)=f_{n}^{n+1} g_{n+1}\left(x_{n+1}\right),
$$

Lemma 4.1(b) gives that $g_{n}=\mu_{n}^{n+1}\left(g_{n+1}\right)$. Thus $g=\left(g_{1}, g_{2}, \ldots\right) \in G_{\infty}$, and from the definition of the action of $G_{\infty}, g(x)=x^{\prime}$.

Now we wish to introduce the (common) universal covering space of the spaces $X_{1}, X_{2}, \cdots$. This step is essential to carrying out our program. Recall that we have fixed a base point $b=\left(b_{1}, b_{2}, \ldots\right)$ in $X_{\infty}$. Let $p_{1}:(\widetilde{X}, \tilde{b}) \rightarrow\left(X_{1}, b_{1}\right)$ be the universal covering space of $X_{1}$ (which exists since $X_{1}$ is nice). Assuming the covering map $p_{n}:(\tilde{X}, \tilde{b}) \rightarrow\left(X_{n}, b_{n}\right)$ has been defined, let $p_{n+1}$ : $(\widetilde{X}, \tilde{b}) \rightarrow\left(X_{n+1}, b_{n+1}\right)$ be the unique covering map which satisfies the relation $f_{n}^{n+1} p_{n+1}=p_{n}$. Because of this relation, the sequence $\left(p_{1}, p_{2}, \cdots\right)$, so defined, induces a map $p_{\infty}:(\widetilde{X}, \tilde{b})=\left(X_{\infty}, b\right)$; explicitly, $p_{\infty}(\tilde{x})=\left(p_{1}(x), p_{2}(x), \cdots\right)$. In the case of the solenoids, $\widetilde{X}$ is a line that $p_{\infty}$ maps continuously and 1-1 onto a path component of $X_{\infty}$, which is dense in $X_{\infty}$. (This follows from general results proved below.) 
Now let $Y_{\infty}=G_{\infty} \times \widetilde{X}$ and define $P_{\infty}: Y_{\infty} \rightarrow X_{\infty}$ by $P_{\infty}(g, \tilde{x})=g p_{\infty}(\tilde{x})$. Call a subset $\widetilde{V}$ of $\widetilde{X}$ simple if $p_{1}$ maps $\widetilde{V}$ homeomorphically onto $p_{1}(\widetilde{V})$. The following lemma is used in the next two theorems.

LEMma 5.4. $P_{\infty}$ is a local homeomorphism of $Y_{\infty}$ onto $X_{\infty}$. In fact, if $\widetilde{V}$ is any simple open subset of $\widetilde{X}$, then $P_{\infty}$ maps $G_{\infty} \times \widetilde{V}$ homeomorphically onto $f_{1}^{-1}\left(p_{1}(\widetilde{V})\right)$.

Proof. To save a duplication, we first prove the following

Sublemma. For any $n$ let $g_{n}^{0} \in G_{n}$, let $W=\mu_{n}^{-1}\left(g_{n}^{0}\right)$, and let $\widetilde{V}$ be an open subset of $\widetilde{X}$. Then $P_{\infty}(W \times \widetilde{V})=f_{n}^{-1}\left[g_{n}^{0} p_{n}(\widetilde{V})\right]$, which is an open set.

Proof. The latter set is open since $p_{n}$ is open. To show the equality suppose that $x \in f_{n}^{-1}\left[g_{n}^{0} p_{n}(\widetilde{V})\right]$. Then $x_{n}=g_{n}^{0} p_{n}(\tilde{v})$ where $\tilde{v} \in \widetilde{V}$. Since then $x_{1}=$ $f_{1}^{n} g_{n}^{0} p_{n}(\tilde{v})=f_{1}^{n} p_{n}(\widetilde{v})=p_{1}(\widetilde{v})$, there is by Lemma 5.3 a unique $g$ in $G_{\infty}$ such that $x=g p_{\infty}(\tilde{v})=P_{\infty}(g, \tilde{v})$. But then $g_{n} p_{n}(\tilde{v})=x_{n}=g_{n}^{0} p_{n}(\tilde{v})$, so that $g_{n}=$ $g_{n}^{0}$, which means that $g \in W$. Therefore $x \in P_{\infty}(W \times \widetilde{V})$. The reverse inclusion is obvious.

From the Sublemma it follows that $P_{\infty}$ is an open map, since sets of the form $W \times \widetilde{V}$ form a basis for the open sets of $Y_{\infty}$.

Now let $\widetilde{V}$ be a simple open subset of $\widetilde{X}$. In the Sublemma take $n=1$. $\left(G_{1}\right.$ consists of the identity element alone.) Then we have $P_{\infty}\left(G_{\infty} \times \widetilde{V}\right)$ $=f_{1}^{-1}\left[p_{1}(\widetilde{V})\right]$. Furthermore, $P_{\infty}$ is $1-1$ on $G_{\infty} \times \widetilde{V}$. For suppose $P_{\infty}(g, \tilde{v})$ $=P_{\infty}\left(g^{\prime}, \widetilde{v}^{\prime}\right)$. Then $p_{1}(\widetilde{v})=p_{1}\left(\widetilde{v}^{\prime}\right)$, and since $p_{1}$ is $1-1$ on $\widetilde{V}, \widetilde{v}=\widetilde{v}^{\prime}$. From the uniqueness part of Lemma $5.3, g=g^{\prime}$. Finally, since $P_{\infty}$ is open, $P_{\infty}$ is a homeomorphism on $G_{\infty} \times \widetilde{V}$. I

THEOREM 5.5. $Y_{\infty}$ is a generalized covering space of $X_{\infty}$ with respect to the $\operatorname{map} P_{\infty}$.

Note. We use the definition in $\mathrm{Hu}[9, \mathrm{p} .104]$. Thus we must show that there is an open covering of $X_{\infty}$ by sets $V$ such that $P_{\infty}^{-1}(V)$ can be represented as a disjoint union of open subsets of $Y_{\infty}$, each of which is mapped homeomorphically onto $V$ by $P_{\infty}$.

Proof. Let $V_{1}$ be an open set in $X_{1}$ such that $p_{1}^{-1}\left(V_{1}\right)$ is the disjoint union $U \widetilde{V}^{i}$ of open sets in $\widetilde{X}$ such that for each $i, p_{1}$ maps $\widetilde{V}^{i}$ homeomorphically onto $V_{1}$. Let $V=f_{1}^{-1}\left(V_{1}\right)$. Now by Lemma $5.4, P_{\infty}$ maps each $G_{\infty} \times \widetilde{V}^{i}$ homeomorphically onto $V$. Furthermore, $\bigcup\left(G_{\infty} \times \widetilde{V}^{i}\right)$ is a disjoint union of open sets in $Y_{\infty}$, which we claim is all of $P_{\infty}^{-1}(V)$. For if $(g, \tilde{x}) \in P_{\infty}^{-1}(V)$, then $p_{1}(\tilde{x}) \in V_{1}$, so that $\tilde{x} \in \widetilde{V}^{i}$ for some $i$. It is obvious that the collection of such sets $V$ covers $X_{\infty}$. I

Theorem 5.6. $\left(X_{\infty}, f_{1}, X_{1}, G_{\infty}\right)$ is a principal fibre bundle.

Proof. The three properties (a), (b), (c) established below can be seen to be equivalent to those in Steenrod's [12] definition of a principal fibre 
bundle; except that, since we have the group $G_{\infty}$ acting on the left of $X_{\infty}$, $G_{\infty}$ will have to act on the right of the fibre ( $G_{\infty}$ itself) in Steenrod's definition. (a) $G_{\infty}$ acts on $X_{\infty}$ as a topological transformation group, as has been shown in Lemma 5.2. (b) $\left(X_{\infty}, f_{1}, X_{1}\right)$ is a locally trivial fibre space with fibre $G_{\infty}$. Let $\widetilde{V}$ be a simple open subset of $\widetilde{X}$ and let $p_{1}(\widetilde{V})=V_{1}$. Such sets $V_{1}$ can be taken as coordinate neighborhoods in $X_{1}$. Let $q: V_{1} \rightarrow \widetilde{V}$ be the inverse of $p_{1} \mid \widetilde{V}$. The map $(g, v) \rightarrow(g, q(v))$ is then a homeomorphism of $G_{\infty} \times V_{1}$ onto $G_{\infty} \times \widetilde{V}$. Thus by Lemma 5.4, the map $\phi: G_{\infty} \times V_{1} \rightarrow f_{1}^{-1}\left(V_{1}\right)$ given by $\phi(g, v)=P_{\infty}(g, q(v))$ is a homeomorphism. And for every $(g, v)$ in $G_{\infty} \times V_{1}$ we have $f_{1} \phi(g, v)=f_{1} g p_{\infty} q(v)=p_{1} q(v)=v$. (c) Finally, the action of $G_{\infty}$ is compatible with the fibre space structure, for we have for any $g$ and $g^{\prime}$ in $G_{\infty}$ and any $v$ in $V_{1}$ that $\phi\left(g g^{\prime}, v\right)=P_{\infty}\left(g g^{\prime}, q(v)\right)=\left(g g^{\prime}\right) p_{\infty} q(v)$ $=g\left[g^{\prime} p_{\infty} q(v)\right]=g P_{\infty}\left(g^{\prime}, q(v)\right)=g \phi\left(g^{\prime}, v\right)$. I

Corollary 5.7. $X_{\infty}$ is not locally connected at any point.

Proof. By Theorem 5.6 and Lemma 5.1, $X_{\infty}$ is locally like a product of a totally disconnected, perfect space with another space. I

In order to understand the structure of $X_{\infty}$ more, we now study its path components. For this the maps $p_{\infty}$ and $P_{\infty}$ will be useful.

Theorem 5.8. Let $K$ denote the subset $p_{\infty}(\widetilde{X})$ of $X_{\infty}$. A subset of $X_{\infty}$ is a path component of $X_{\infty}$ if and only if it is a "translate" of $K$ by some element $g$ of $G_{\infty}$, i.e., it is of the form $g(K)$. Each path component of $X_{\infty}$ is dense in $X_{\infty}$.

Proof. Let us take a translate $g(K)$ and prove it is a path component. First of all, $g(K)$ is pathwise connected since it is a continuous image of the pathwise connected space $\widetilde{X}$. Suppose $\alpha$ is a path from a point $x$ in $g(K)$ to a point $y$ in $\underset{\sim}{X}$. We must show that $y$ also is in $g(K)$. Now $x=g p_{\infty}(\tilde{x})$ for some $\tilde{x}$ in $\widetilde{X}$; that is, $(g, \tilde{x})$ lies over $x$ with respect to the generalized covering map $P_{\infty}$ (Theorem 5.5). Thus we may lift $\alpha$ by $P_{\infty}$ to a path $\tilde{\alpha}$ in $Y_{\infty}$ starting at $(g, \tilde{x})$. But $\{g\} \times \widetilde{X}$ is a component of $Y_{\infty}$, and since $\tilde{\alpha}$ begins in this component, it must end in it. Thus $\tilde{\alpha}(1)$ is of the form $(g, \tilde{y})$, and we have $g p_{\infty}(\tilde{y})=P_{\infty}(g, \tilde{y})=P_{\infty} \tilde{\alpha}(1)=\alpha(1)=y$, so that $y \in g(K)$.

Thus the sets $g(K)$ are path components. They exhaust all of $X_{\infty}$, for by Theorem 5.5, $P_{\infty}$ maps $Y_{\infty}$ onto $X_{\infty}$.

To show that the sets $g(K)$ are dense in $X_{\infty}$, it is sufficient to show that $K$ is dense. Open sets of the form $V=f_{n}^{-1}\left(V_{n}\right)$ where $V_{n}$ is open in $X_{n}$ form a basis for the open sets in $X_{\infty}$. Take any such $V_{n}$. Since $p_{n}: \widetilde{X} \rightarrow X_{n}$ is onto, there is a point $\tilde{v}$ in $\widetilde{X}$ for which $p_{n}(\tilde{v}) \in V_{n}$. Then $p_{\infty}(\tilde{v}) \in K \cap V$. I

The path components $g(K)$ are of course disjoint if they are different, but there can be $g \neq g^{\prime}$ such that $g(K)=g^{\prime}(K)$. We will determine when this can happen. Since $G_{\infty}$ is a group, $g(K)=g^{\prime}(K)$ is equivalent to $g^{-1} g^{\prime}(K)=K$. So we will determine which $g$ are such that $g(K)=K$. In this direction we let $H$ denote the covering transformation group of $p_{1}: \widetilde{X} \rightarrow X_{1}$, 
and we define a certain homomorphism $\sigma: H \rightarrow G_{\infty}$. It will turn out that $g(K)=K$ if and only if $g$ is in the image of $\sigma$. Also the kernel of $\sigma$ has significance (Theorems 5.12 and 5.13).

Let $\lambda: F_{1} \rightarrow H$ be the canonical isomorphism. (with respect to the base point $\widetilde{b} \in \widetilde{X})$. For each $n$ let $\tau_{n}: F_{1} \rightarrow Q_{n}=F_{1} / F_{n}$ be the natural homomorphism. Since $\nu_{n}^{n+1} \tau_{n+1}=\tau_{n}$, the $\tau_{n}$ 's induce a homomorphism $\tau: F_{1} \rightarrow Q_{\infty}$ given by $\tau(a)=\left(\tau_{1}(a), \tau_{2}(a), \cdots\right)$. Now define $\sigma$ by commutativity in the diagram

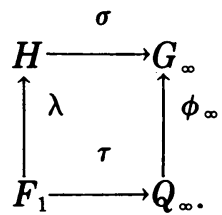

LEMma 5.9. For each $h$ in $H$ the following diagram is commutative:

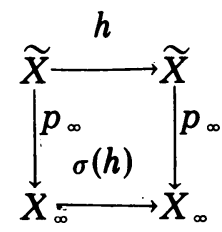

Proof. Let $g=\sigma(h)$. Proving that $g p_{\infty}=p_{\infty} h$ is equivalent to proving that for each $n, g_{n} p_{n}=p_{n} h$ (where $g_{n}=\mu_{n}(g)$ ). To show the latter we use Lemma 4.1. In the preliminary discussion of that section replace diagram (4.1) by the commutative diagram

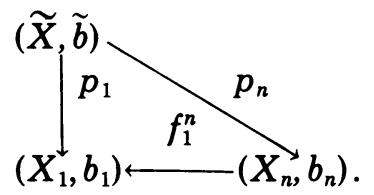

Then diagram (4.2) gets replaced by the diagram

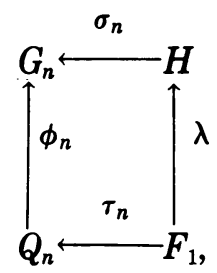

where, according to $\S 4, \sigma_{n}$ is defined by commutativity in this diagram. Then by Lemma $4.1, \sigma_{n}(h) p_{n}=p_{n} h$. Thus we need only show that $g_{n}=\sigma_{n}(h)$. But $g_{n}=\mu_{n}(g)=\mu_{n} \sigma(h)=\mu_{n} \phi_{\infty} \tau \lambda^{-1}(h)=\phi_{n} \nu_{n} \tau \lambda^{-1}(h)=\phi_{n} \tau_{n} \lambda^{-1}(h)=\sigma_{n}(h)$. |

Theorem 5.10. For $g \in G_{\infty}, g(K)=K$ if and only if $g=\sigma(h)$ for some $h \in H$. 
Proof. Suppose first $g=\sigma(h)$. Then for each $\tilde{x}$ in $\widetilde{X}$, by the preceding lemma, $g p_{\infty}(\tilde{x})=p_{\infty} h(\tilde{x}) \in K$ so that $g(K) \subset K$, whence $g(K)=K$. Conversely, suppose $g(K)=K$. Then for some $\tilde{x}$ and $\tilde{y}$ in $\widetilde{X}$ we have $g p_{\infty}(\tilde{x})$ $=p_{\infty}(\tilde{y})$. Then since $p_{1}(\tilde{x})=p_{1}(\tilde{y})$, there is an $h$ in $H$ such that $h(\tilde{x})=\tilde{y}$. But then $g p_{\infty}(\tilde{x})=p_{\infty}(\tilde{y})=p_{\infty} h(\tilde{x})=\sigma(h) p_{\infty}(\tilde{x})$. Thus $g$ and $\sigma(h)$ are elements of $G_{\infty}$ which agree on a point, so they are equal. I

Corollary 5.11. If the fundamental group $F_{1}$ of $X_{1}$ is countable (in particular if $X_{1}$ is a polyhedron) then $X_{\infty}$ has uncountably many path components.

Proof. Let $S$ be the set of path components of $X_{\infty}$. Define a map $w$ from the space $G_{\infty} / \sigma(H)$ of left cosets of $\sigma(H)$ to $S$ by $w(g \sigma(H))=g(K)$. By the preceding theorem, $w$ is well defined and 1-1; by Theorem $5.8, w$ is onto. Now $G_{\infty}$ is uncountable since each bonding map $\mu_{n}^{n+1}: G_{n+1} \rightarrow G_{n}$ has everywhere nondegenerate point inverses. And since $\sigma(H)=\sigma \lambda\left(F_{1}\right)$ is countable, $G_{\infty} / \sigma(H)$ is uncountable. I

In the cases of solenoids the map $p_{\infty}$ of $\widetilde{X}$ onto $K$ is $1-1$. What is the general situation? This question is answered by the following theorem.

Theorem 5.12. (a) The map $p_{\infty}: \widetilde{X} \rightarrow X_{\infty}$ is $1-1$ if and only if the homomorphism $\sigma: H \rightarrow G_{\infty}$ is 1-1. (b) The kernel of $\tau: F_{1} \rightarrow Q_{\infty}$ is $N=\bigcap_{n=1}^{\infty} F_{n}$. (c) Hence $p_{\infty}$ is 1-1 if and only if $N=1$.

Proof. (a) Suppose $p_{\infty}$ is 1-1. Then $\sigma$ must be $1-1$, for if $\sigma(h)=1$, we have by Lemma 5.9 that for all $\tilde{x}$ in $\widetilde{X}, p_{\infty} h(\tilde{x})=\sigma(h) p_{\infty}(\tilde{x})=p_{\infty}(\tilde{x})$. Hence $h(\tilde{x})=\tilde{x}$, and $h=1$. Conversely, suppose $\sigma$ is $1-1$ and suppose $p_{\infty}(\tilde{x})$ $=p_{\infty}(\tilde{y})$. Then since $p_{1}(\tilde{x})=p_{1}(\tilde{y})$ there is an $h$ in $H$ such that $h(\tilde{x})=\tilde{y}$; therefore $p_{\infty}(\tilde{x})=p_{\infty}(\tilde{y})=p_{\infty} h(\tilde{x})=\sigma(h) p_{\infty}(\tilde{x})$. Since $\sigma(h)$ is in $G_{\infty}$ and has a fixed point, it must be the identity. Thus $h=1$, and $\tilde{x}=h(\tilde{x})=\tilde{y}$.

(b) Suppose $a \in \operatorname{ker} \tau$. This means that $\tau(a)=\left(a F_{1}, a F_{2}, \cdots\right)=\left(F_{1}, F_{2}, \cdots\right)$ so that $a \in F_{n}$ for each $n$, and $a \in N$. The converse is then obvious.

(c) In diagram (5.1) $\lambda$ and $\phi_{\infty}$ are isomorphisms. Hence $\operatorname{ker} \sigma=\lambda(N)$. The statement follows from (a) and (b). I

TheOREm 5.13. The fundamental group of each path component of $X_{\infty}$ is isomorphic to $N=\bigcap_{n=1}^{\infty} F_{n}$.

Proof. By Theorem 5.8 all path components are homeomorphic. We will show that for the map $f_{1}:\left(X_{\infty}, b\right) \rightarrow\left(X_{1}, b_{1}\right), \eta=\left(f_{1}\right)_{*}$ takes

$$
\pi(K, b)=\pi\left(X_{\infty}, b\right)
$$

isomorphically onto $N$. Since $f_{1}=f_{1}^{n} f_{n}$, the range of $\eta$ is in $N$.

To prove that the range of $\eta$ is all of $N$, suppose $\left[\alpha_{1}\right] \in N$. Since $\left[\alpha_{1}\right] \in F_{n}$, the lifting of $\alpha_{1}$ by $f_{1}^{n}$ to the path $\alpha_{n}$ starting at $b_{n}$ is a loop on $b_{n}$. And since then $f_{n}^{n+1} \alpha_{n+1}=\alpha_{n}$, we may define a loop $\alpha$ on $b$ by $\alpha(t)=\left(\alpha_{1}(t), \alpha_{2}(t), \cdots\right)$, for which obviously $\eta([\alpha])=\left[\alpha_{1}\right]$. To show that $\eta$ is $1-1$, suppose $\eta([\alpha])=1$. 
This means that there is a homotopy $h_{t}^{1}$ of loops on $b_{1}$ such that $h_{0}^{1}=\alpha_{1}$ and $h_{1}^{1}=b_{2}$. Applying successively the covering homotopy theorem to the maps $f_{n}^{n+1}$ we may construct a sequence $\left(h_{t}^{n}\right)$ such that for each $n$, (1) $h_{t}^{n}$ is a homotopy of loops on $b_{n}$ in $X_{n}$, (2) $h_{0}^{n}=\alpha_{n}$, (3) $h_{1}^{n}=b_{n}$, and (4) $f_{n}^{n+1} h_{t}^{n+1}$ $=h_{t}^{n}$. This sequence then induces a homotopy $h_{t}$ of loops on $b$ in $X_{\infty}$ such that $h_{0}=\alpha$ and $h_{1}=b$. I

REMARK 5.14. If $(X, f)$ is an inverse limit sequence where each map $f_{1}^{n}$ is a covering map, but is not necessarily regular, the proof above shows that for every $x$ in $X_{\infty}$, if $K_{x}$ denotes the path component of $x$, then $\pi\left(K_{x}, x\right)$ $\approx \bigcap_{n=1}^{\infty}\left(f_{1}^{n}\right)_{*}\left(\pi\left(X_{n}, x_{n}\right)\right)$.

6. Homogeneity of certain solenoidal spaces. Let us call a space $X$ pathhomogeneous if for every pair of points $x, y$ in $X$ there is a path $\alpha$ from $x$ to $y$ and a homeomorphism $h$ of $(X, x)$ onto $(X, y)$ such that $h$ induces the same isomorphism of $\pi(X, x)$ onto $\pi(X, y)$ as the path $\alpha$ does.

Theorem 6.1. If $(X, f)$ is a solenoidal sequence for which $X_{1}$ is path-homogeneous, then the solenoidal space $X_{\infty}$ is homogeneous.

Proof. Let us use the notation of the preceding section. Suppose $x$ and $y$ are points in $X_{\infty}$. We wish to show that there is alhomeomorphism of $\left(X_{\infty}, x\right)$ onto $\left(X_{\infty}, y\right)$. Since $X_{1}$ is path-homogeneous there is a path $\alpha_{1}$ from $x_{1}$ to $y_{1}$ and a homeomorphism $h_{1}:\left(X_{1}, x_{1}\right) \rightarrow\left(X_{1}, y_{1}\right)$ such that $\left(h_{1}\right)_{*}=\left(\alpha_{1}\right)_{*}$ : $\pi\left(X_{1}, x_{1}\right) \rightarrow \pi\left(X_{1}, y_{1}\right)$; that is, $\left(h_{1}\right)_{*}([\gamma])=\left[h_{1} \gamma\right]=\left[\alpha_{1}^{-1} \cdot \gamma \cdot \alpha_{1}\right]=\left(\alpha_{1}\right)_{*}([\gamma])$ for all $[\gamma] \in \pi\left(X_{1}, x_{1}\right)$. Now lift $\alpha_{1}$ by $p_{1}$ to some path $\tilde{\alpha}$ in $\widetilde{X}$. Let $u$ $=p_{\infty}(\tilde{\alpha}(0))$ and $v=p_{\infty}(\tilde{\alpha}(1))$. Since $u_{1}=x_{1}$ there is, by Lemma 5.3 , a (unique) element $g$ of $G_{\infty}$ such that $g(u)=x$. (In particular $g$ is a homeomorphism of $\left(X_{\infty}, u\right)$ onto $\left(X_{\infty}, x\right)$.) Similarly there is a $g^{\prime}$ in $G_{\infty}$ such that $g^{\prime}(v)$ $=y$. If we can find a homeomorphism $h$ of $\left(X_{\infty}, u\right)$ onto $\left(X_{\infty}, v\right)$ we will be through, since then $g^{\prime} h g^{-1}$ takes $x$ onto $y$.

We will construct such an $h$ from $h_{1}$ by a lifting process which makes use of the path $\tilde{\alpha}$. Let $\alpha_{n}=p_{n} \tilde{\alpha}$; this path goes from $u_{n}$ to $v_{n}$. Observe that $f_{n}^{n+1} \alpha_{n+1}=f_{n}^{n+1} p_{n+1} \tilde{\alpha}=p_{n} \tilde{\alpha}=\alpha_{n}$. Let $\psi_{n}: \pi\left(X_{n}, u_{n}\right) \rightarrow \pi\left(X_{n}, v_{n}\right)$ denote the isomorphism induced by $\alpha_{n}$. We claim that the following diagram is commutative.

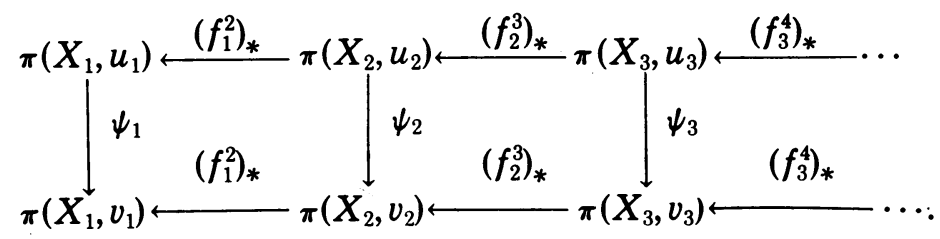


For suppose $[\gamma] \in \pi\left(X_{n+1}, u_{n+1}\right)$. Then

$$
\begin{aligned}
\left(f_{n}^{n+1}\right)_{*} \psi_{n+1}([\gamma]) & =\left(f_{n}^{n+1}\right)_{*}\left[\alpha_{n+1}^{-1} \cdot \gamma \cdot \alpha_{n+1}\right] \\
& =\left[\left(f_{n}^{n+1} \alpha_{n+1}\right)^{-1} \cdot f_{n}^{n+1} \gamma \cdot f_{n}^{n+1} \alpha_{n+1}\right] \\
& =\left[\alpha_{n}^{-1} \cdot f_{n}^{n+1} \gamma \cdot \alpha_{n}\right]=\psi_{n}\left(f_{n}^{n+1}\right)_{*}([\gamma]) .
\end{aligned}
$$

Looking at the first rectangle in (6.1), using what $\mathrm{Hu}[9$, p.90] calls the fibre map theorem, and recalling that $\left(h_{1}\right)_{*}=\psi_{1}$, we have a unique map

$$
h_{2}:\left(X_{2}, u_{2}\right) \rightarrow\left(X_{2}, v_{2}\right)
$$

which makes the first rectangle in (6.2) below commutative. Furthermore, $h_{2}$ is a homeomorphism. For, using $\psi_{1}^{-1}$ and $\psi_{2}^{-1}$, there is a (unique) map $h_{2}^{\prime}:\left(X_{2}, v_{2}\right) \rightarrow\left(X_{2}, u_{2}\right)$ such that $f_{1}^{2} h_{2}^{\prime}=h_{1}^{-1} f_{1}^{2}$. But then $f_{1}^{2} h_{2}^{\prime} h_{2}=h_{1}^{-1} f_{1}^{2} h_{2}$ $=h_{1}^{-1} h_{1} f_{1}^{2}=f_{1}^{2}$, and from the uniqueness of liftings, $h_{2}^{\prime} h_{2}=$ identity. Similarly $h_{2} h_{2}^{\prime}=$ identity. Now since $\left(f_{1}^{2}\right)_{*}$ is a monomorphism, $\left(h_{2}\right)_{*}=\psi_{2}$. Thus we can use the second rectangle of (6.1) to get a homeomorphism $h_{3}$ making the second rectangle of (6.2) commutative. We continue this process, obtaining $\left(h_{1}, h_{2}, h_{3}, \cdots\right)$.

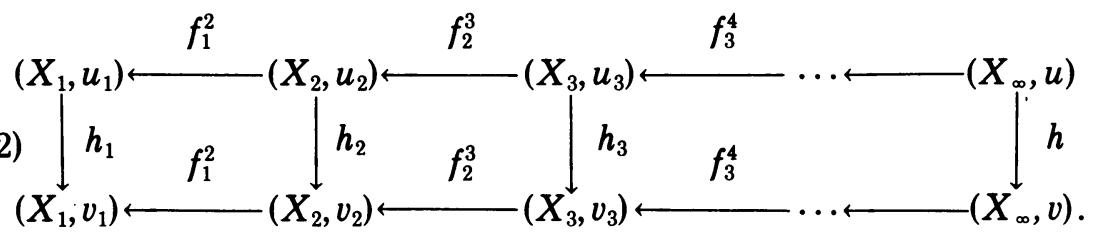

Clearly the induced map $h$ is the desired homeomorphism. I

Let us use $n$-manifold in the very general sense of a connected Hausdorff space, each of whose points is contained in an open set homeomorphic to euclidean $n$-space $E^{n}$.

LEMMA 6.2. Every n-manifold is path-homogeneous.

CoRollary 6.3. If $(X, f)$ is a solenoidal sequence where $X_{1}$ is an n-manifold, then $X_{\infty}$ is homogeneous.

Proof of Lemma 6.2. By an isotopic deformation $h_{t}$ of a space $S$ let us mean an isotopy $h_{t}: S \rightarrow S$ such that $h_{0}=$ identity.

Let $M$ be an $n$-manifold. First suppose $U$ is an open subset of $M$ for which there is a homeomorphism $g: U \rightarrow E^{n}$, and let $z, w \in U$. Let $\{g(z), g(w)\}$ $\subset\left\{x \in E^{n}:\|x\|<r\right\}$. It is easy to see that there is an isotopic deformation $h_{t}^{\prime}$ of $E^{n}$ such that $h_{i}^{\prime}(g(z))=g(w)$ and for each $t, h_{t}^{\prime}$ is the identity on

$$
\left\{x \in E^{n}:\|x\| \geqq r\right\} \text {. }
$$


Define $h_{t}: M \rightarrow M$ by $h_{t}(m)=g^{-1} h_{t}^{\prime} g(m)$ for $m \in U$ and $h_{t}(m)=m$ for $m \notin U$. Using the fact that $M$ is Hausdorff one can see that $h_{t}$ is an isotopic deformation of $M$; and $h_{1}(z)=w$.

Now let $x$ and $y$ be arbitrary points of $M$. Since $M$ is connected, there is a chain of open sets $\left(U_{1}, U_{2}, \cdots, U_{r}\right)$, each homeomorphic to $E^{n}$, such that $x \in U_{1}$ and $y \in U_{r}$. For each $i=2,3, \cdots, r$ choose $x_{i} \in U_{i-1} \cap U_{i}$, and let $x_{1}=x, x_{r+1}=y$. By the preceding paragraph there is for each $i=1,2, \cdots$, $r$ an isotopic deformation $h_{t}^{i}$ of $M$ such that $h_{1}^{i}\left(x_{i}\right)=x_{i+1}$. Then the composition $h_{t}=h_{t}^{r} \ldots h_{t}^{2} h_{t}^{1}$ is an isotopic deformation of $M$ such that $h_{1}(x)=y$.

Now it follows from [6, p.57] that the path $\alpha$ from $x$ to $y$ defined by $\alpha(t)=h_{t}(x) \quad(0 \leqq t \leqq 1)$ induces the same isomorphism of $\pi(M, x)$ onto $\pi(M, y)$ as $h_{1}$ does. I

The author suspects that Corollary 6.3 is no longer valid when the bonding maps are nonregular covering maps, even when the $X_{n}$ 's are compact differentiable manifolds. (J. Segal [10] announced in an abstract that if $(X, f)$ is an inverse limit sequence where each $X_{n}$ is a homogeneous, connected, and locally pathwise connected space and where the bonding maps are covering maps, then $X_{\infty}$ is homogeneous. However he informed the author of an error in the proof.) The author has shown that such a counterexample can be constructed if a certain group theoretic construction can be made; and, in turn, this can be done provided a certain sequence of graphs and covering maps can be produced. The idea includes using Theorem 5.13 (see Remark 5.14) to show that the continuum has two nonhomeomorphic path components.

Added in proof: Richard M. Schori (Dissertation, State University of Iowa, 1964) has constructed an inverse limit sequence of compact, orientable 2-manifolds such that the bonding maps are (nonregular) covering maps and such that the limit is not homogeneous.

\section{Bibliography}

1. R. H. Bing, A homogeneous indecomposable plane continuum, Duke Math. J. 15(1948), 729742.

2. , Higher-dimensional hereditarily indecomposable continua, Trans. Amer. Math. Soc. 71(1951), 267-273.

3. A simple closed curve is the only homogeneous bounded plane continuum that contains an arc, Canad. J. Math. 12(1960), 209-230.

4. Morton Brown, On the inverse limit of Euclidean N-spheres, Trans. Amer. Math. Soc. 96(1960), 129-134.

5. J. H. Case, Another 1-dimensional homogeneous continuum which contains an arc, Pacific J. Math. 11(1961), 455-469.

6: R. H. Crowell and R. H. Fox, Introduction to knot theory, Ginn and Co., Boston, Mass., 1963.

7. S. Eilenberg and N. Steenrod, Foundations of algebraic topology, Princeton Univ. Press, Princeton, N. J., 1952. 
8. M. K. Fort, Jr., Homogeneity of infinite products of manifolds with boundary, Topology of 3manifolds, Prentice-Hall, Englewood Cliffs, N. J., 1962.

9. S.-T. Hu, Homotopy theory, Academic Press, New York, 1959.

10. J. Segal, Homogeneity of inverse limit spaces, Notices Amer. Math. Soc. 5(1958), 687.

11. H. Seifert and W. Threlfall, Lehrbuch der Topologie, Teubner, Leipzig, 1934.

12. N. Steenrod, The topology of fibre bundles, Princeton Univ. Press, Princeton, N. J., 1951.

13. D. van Dantzig, Über topologisch homogene Kontinua, Fund. Math. 14(1930), 102-125.

14. A. van Heemert, Topologische Gruppen und unzerlegbare Kontinua, Compositio Math. 5(1938), 319-326.

YALE UNIVERSITY,

New Haven, Connecticut

The University of Georgia, Athens, Georgia 